\title{
Fatores motivadores de uso de site web: um estudo de caso
}

Tatiana de Almeida Furquim

Mestre em ciência da informação pela Universidade de Brasília (UnB). Funcionária do Serviço Federal de Processamento de Dados (Serpro). E-mail: tatiana-almeida.furquim@serpro.gov.br

\section{Resumo}

A partir da adaptação de um conjunto de critérios de avaliação de sites web centrados no usuário, foram identificados fatores motivadores de uso de site de governo eletrônico. O site web selecionado para análise foi o Comprasnet. O objetivo geral da pesquisa foi identificar os critérios determinantes na tomada de decisão pela opção de uso do Comprasnet. Para tanto, foram realizadas entrevistas por telefone, em âmbito nacional, com os usuários do Comprasnet. Concluiu-se com a apresentação e discussão dos fatores motivadores de uso do Comprasnet, e foram apresentadas as limitações e recomendações para estudos posteriores.

\section{Palavras-chave}

Qualidade de site web; Avaliação centrada no usuário; Comprasnet; Sistemas centrados no usuário.

\section{Motivating factors in use of web site: a case study}

\begin{abstract}
Motivating factors for use of electronic government site have been identified from an adaptation of a set of evaluation criteria of web sites focused on the user. The web site selected for analysis was Comprasnet. The general purpose of the research was to identify the predominant criteria in decision-making when Comprasnet was chosen. Several interviews were carried out with Comprasnet users by telephone nationwide. As a conclusion, presentation and discussion of the motivating factors were brought forth, as well as the hampers and recommendations for further studies.
\end{abstract}

\section{Keywords}

Web site quality; User-centered evaluation; Comprasnet; e-GOV; User-centered systems.

\section{INTRODUC̣ÃO}

Este artigo trata de estudo de caso destinado a alcançar um entendimento sobre os fatores relativos a um site web que motivam as pessoas a utilizá-lo. Para tanto, buscou-se embasamento teórico na literatura científica sobre avaliação de sites web e marketing da informação, conforme apresentado resumidamente a seguir.

\section{REFERENCIAL TEÓRICO}

Segundo Kotler (1996, p. 25), "o pensamento de marketing começa com as necessidades e os desejos humanos”. O que faz o ser humano escolher entre as diferentes opções que lhe são oferecidas é ainda uma questão não resolvida, e o marketing configura-se como uma das áreas do conhecimento que se interessam pela compreensão deste fenômeno.

De acordo com o pensamento de marketing, o conhecimento das necessidades, percepções e satisfação dos consumidores permitirá a melhor estruturação da oferta. Esta pesquisa baseia-se neste princípio, ao buscar, junto ao usuário, os critérios considerados por eles ao avaliar um site web.

Lindroos (1997) afirma que uma das grandes diferenças de um site web em relação a um sistema de informação tradicional é justamente a não-existência de um público usuário cativo, como era o caso dos softwares, na sua maioria utilizados por funcionários de uma organização, cujo uso geralmente é determinado de forma imperativa. Para a autora, as técnicas de análise e segmentação de mercado aplicado ao site web que se pretende desenvolver podem auxiliar a resolver esta questão. A autora especifica a necessidade da aplicação dos conhecimentos mercadológicos para o estudo dos sites web.

De acordo com Berners-Lee (1999, p. 124), “em 1996 a maioria do que acontecia na web era levado a cabo por pura excitação", porém a partir de 1998 a web passou a ser vista como um "campo de batalha de grandes negócios e grandes interesses governamentais". Shneiderman (1997) complementa ao asseverar que "refinar a web é mais do que um desafio técnico ou um objetivo comercial, pois cada vez mais o governo passa a oferecer informações e serviços on-line e as instituições educacionais aumentam sua dependência da web". 
Segundo Berghel et alii (1999), há uma base de 100 milhões de sites web, 10 mil são adicionados por mês. Trochim (1996) assevera que, enquanto muitos esforços são despendidos para criar e aumentar o uso da Internet, poucos esforços são conduzidos no sentido de avaliá-la.

Bevan (1998) sugere o uso de listas de verificação como um dos métodos de avaliação de sites web. Todavia, os critérios de avaliação discutidos nas listas de verificação encontradas na literatura cientifica estão longe de atingir um consenso:

"Não apenas existe pouca concordância entre as fontes sobre quais são os critérios mais importantes ou mais apropriados para avaliar um site web, como também poucos desses critérios aparecem em revisões, sugerindo uma falta de consenso continuada" (Sweetland, 2001).

Smith (1997) desenvolveu um trabalho de consolidação dos critérios utilizados por sites web especializados em avaliação, conforme mostra a tabela 1 .

Todavia, permanece a questão: o que pensam os usuários da web? Segundo Rosenfield \& Morvile (1998, p. 8), os usuários da web "detestam" quando não conseguem encontrar no site web a informação que eles sabem que está lá, projeto gráfico pobre, uso gratuito da tecnologia (figuras animadas, sons, filmes que somente tornam o tempo de resposta da página web mais longo, sem agregar valor ao conteúdo), "tom inapropriado" (uso de jargões ou de uma linguagem não-adequada ao usuário), construção da página web centrada nos critérios do produtor, páginas web "em construção" e "falta de atenção aos detalhes" (links que não funcionam, conteúdo desatualizado, falta de datas, programas que executam com erro). Por outro lado, consideram que os usuários são motivados por estética: ou seja, os usuários podem usar um site web somente porque o site web é esteticamente agradável; boas idéias: sites web que mudam a forma como o usuário enxerga as coisas; utilidade: para os autores, os usuários visitam e retornam a um site web que de alguma forma julgam úteis; possibilidade que a web oferece de se poder encontrar praticamente tudo; finalmente, a possibilidade de se poder obter no site web informações customizadas e personalizadas.

Assim, outra forma de conhecer critérios de avaliação de sites web é perguntando diretamente ao usuário o que ele espera de um site web e como o avalia. Abels, White \& Hahn (1997, 1998), com o intuito de construir um site web baseado nos critérios do próprio usuário, identificaram, junto ao público-alvo do site web a ser desenvolvido, quais seriam os critérios que estes
TABELA 1

Freqüência de consideração de critérios em sites web de avaliação

\begin{tabular}{l|c}
\hline Critérios & Número de sites web \\
\hline Gráficos e projeto multimídia & 10 \\
Navegabilidade e organização & 8 \\
Atualidade & 8 \\
Conteúdo (em geral) & 7 \\
Autoridade & 5 \\
Unicidade & 4 \\
Audiência & 4 \\
Funcionalidade (em geral) & 4 \\
Conectividade & 4 \\
\hline
\end{tabular}

Fonte: Adaptado de Smith (1997)

considerariam os mais importantes quando fossem avaliá-lo. Por estar alinhado às premissas desta pesquisa, o estudo realizado pelas autoras serviu de base para o desenvolvimento deste trabalho.

\section{MÉTODO}

A pesquisa, de caráter exploratório, foi realizada mediante um estudo de caso. O caso analisado foi o Comprasnet, que implementa funcionalidades de governo eletrônico. O Serviço Federal de Processamento de Dados (Serpro) mantém uma central de atendimento ao usuário (CAS) acessível a partir de ligação telefônica gratuita. A base de dados da CAS foi utilizada para delimitar o universo da pesquisa. Em levantamento realizado em agosto de 2002, o número de usuários do Comprasnet registrados foi 2.719. Para a realização da pesquisa, que trabalhou com amostra aleatória, nível de confiança de $99 \%$ e a margem de erro admitida de $4 \%$, a partir da fórmula apresentada por Richardson (1999, p. 171), foi identificado o valor mínimo de 928 respostas dos usuários. A coleta de dados foi operacionalizada por meio de entrevistas via telefone, realizadas pelos técnicos do Serpro, durante a segunda quinzena de agosto de 2002. Foram entrevistados 954 usuários do Comprasnet, distribuídos por todo o território nacional.

\section{RESULTADOS}

Diferentes aspectos devem ser considerados ao analisar freqüências de uso de sites web, como, por exemplo, a quantidade de acessos e a freqüência de atualização. O perfil dos usuários entrevistados foi definido em relação à freqüência e tempo de uso do Comprasnet. Para o Comprasnet, que apresenta média de $147 \mathrm{mil}$ acessos mensais e freqüência diária de atualização, acessos de mais de uma vez na semana foram considerados como 
alta freqüência, enquanto apenas um acesso semanal e acessos quinzenais representaram freqüência média, e acessos mensais, baixa freqüência de uso, conforme ilustrado na figura 1.

O tempo de uso de um site web também pode ser um indicativo da autoridade da opinião do usuário, complementando a análise dos dados referentes à freqüência de uso, partindo-se do pressuposto de que o conhecimento sobre o site web influencia a autoridade da opinião do usuário, que poderá aumentar à medida que o site web for utilizado ao longo do tempo. Assim, embora um usuário possa declarar que acessa o site web diariamente (alta freqüencia de uso), caso o tempo de uso seja muito baixo (inferior a uma semana, por exemplo), é aconselhável questionar a autoridade da opinião deste usuário. O tempo de uso do Comprasnet, de acordo com a amostra pesquisada, distribui-se conforme ilustrado na figura 2 .

A tabela 2 apresenta os fatores motivadores de uso do Comprasnet ordenados em termos de sua freqüência de ocorrência, em ordem decrescente.

\section{ANÁLISE DOS RESULTADOS}

Ao ponderar sobre a evolução das exigências do usuário da web, Nielsen (1997) afirma que o usuário busca "valor agregado" ao utilizar um site web, ou seja, "o usuário busca na web a resolução de um problema”. Todavia, esta definição não está limitada à busca da resolução de um problema "informacional", pois a web oferece a possibilidade de automatização completa de processos de negócios, conforme se pode verificar no caso em questão: o Comprasnet automatiza um dos processos de compras do governo federal, possibilitando a realização de um processo de negócios via web. Assim, o usuário tem a possibilidade de participar de todo o processo licitatório e, inclusive, finalizar uma venda para o governo federal de forma on-line.

Portanto, o "valor agregado" do Comprasnet pode estar intimamente ligado à utilidade da informação percebida pelo seu usuário, conforme foi verificado ao se analisar a explicação dos respondentes que apresentaram "outros motivos" de influência na opção de uso, como, por exemplo, o número de usuários que definiram que utilizam o Comprasnet para consultar licitações do governo federal e participar de pregões eletrônicos, que foram as respostas mais freqüentes entre os usuários que alegaram "outros

\section{FIGURA 1}

Freqüência de uso do Comprasnet

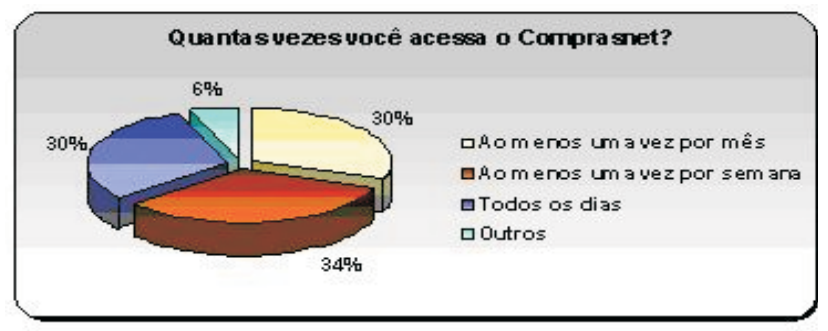

FIGURA 2

Tempo de uso do Comprasnet

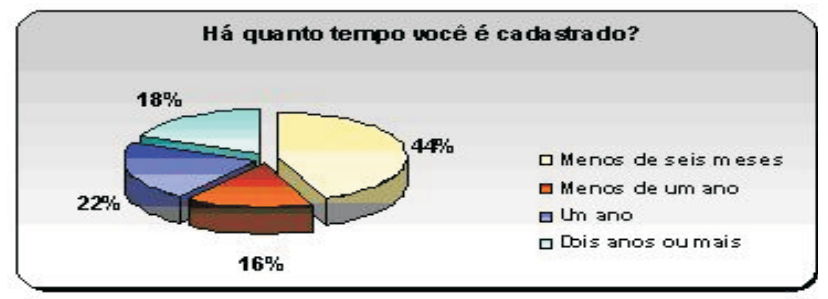

TABELA 2

Fatores motivadores de uso do Comprasnet

\begin{tabular}{|c|c|c|c|}
\hline Categoria & Fator & Freqüência & Posição \\
\hline Conteúdo & Utilidade da informação & $89 \%(n=849)$ & 1 \\
\hline Conteúdo & Confiabilidade da informação & $88 \%(n=840)$ & 2 \\
\hline Estrutura & $\begin{array}{l}\text { Facilidade de entendimento da } \\
\text { estrutura do site web }\end{array}$ & $84 \%(n=799)$ & 3 \\
\hline Aparência & Efeito visual agradável & $84 \%(n=799)$ & 3 \\
\hline Conteúdo & Atualidade da informação & $83 \%(n=793)$ & 4 \\
\hline Aparência & Impressão satisfatória das telas & $82 \%(n=786)$ & 5 \\
\hline Links & Coerência dos links & $77 \%(n=738)$ & 6 \\
\hline Links & Inexistência de links quebrados & $74 \%(n=703)$ & 7 \\
\hline Uso & Facilidade de uso & $67 \%(n=638)$ & 8 \\
\hline Estrutura & $\begin{array}{l}\text { Facilidade de localização da } \\
\text { informação desejada }\end{array}$ & $63 \%(n=604)$ & 9 \\
\hline Conteúdo & $\begin{array}{l}\text { Comodidade para encontrar } \\
\text { a informação }\end{array}$ & $62 \%(n=596)$ & 10 \\
\hline \multicolumn{4}{|c|}{ Centro de gravidade da amostra } \\
\hline Uso & Tempo de resposta & $56 \%(n=533)$ & 11 \\
\hline Pesquisa & $\begin{array}{l}\text { Existência de mecanismo de } \\
\text { "busca no site" }\end{array}$ & $48 \%(n=455)$ & 12 \\
\hline Uso & Existência de mapa do site & $45 \%(n=432)$ & 13 \\
\hline Uso & Facilidade de navegação & $42 \%(n=403)$ & 14 \\
\hline Pesquisa & $\begin{array}{l}\text { Efetividade do mecanismo } \\
\text { de busca }\end{array}$ & $40 \%(n=380)$ & 15 \\
\hline Aparência & $\begin{array}{l}\text { Inibição de figuras sem perda da } \\
\text { funcionalidade }\end{array}$ & $29 \%(n=272)$ & 16 \\
\hline Outra & $\begin{array}{l}\text { Outros fatores determinantes } \\
\text { de uso }\end{array}$ & $29 \%(n=271)$ & 16 \\
\hline Estrutura & Ausência de textos longos & $27 \%(n=259)$ & 17 \\
\hline Aparência & $\begin{array}{l}\text { Utilização não excessiva de } \\
\text { ilustração e efeitos visuais }\end{array}$ & $23 \%(n=217)$ & 18 \\
\hline
\end{tabular}


fatores" como motivadores de sua opção pelo uso. Além disso, outros autores defendem que a qualidade da informação é a principal determinante do uso de um site web. Assim, pode-se asseverar que os dados aqui apresentados corroboram as afirmações de Lindroos (1997) e Nielsen (1997), que concordam que o valor agregado da informação, produto ou serviço oferecido pelo software é a principal motivação do seu uso, e não vice-versa.

Em termos de confiabilidade da informação, importa lembrar Harris (1997), que define o critério confiabilidade da informação como a "autoridade da fonte percebida pelo usuário", o que parece ser confirmado pelos usuários do Comprasnet. Todavia, ao se tratar o conceito de confiabilidade em meio on-line, surgem outras preocupações sobre segurança da informação, em diferentes níveis. Estes aspectos relacionam-se com a confiabilidade da informação, porém não mais apenas no sentido da autoridade da fonte, como também na sensação de segurança que é oferecida ao usuário pelo publicador e mantenedor do site web, no caso do Comprasnet, o Serpro. Esta pode ser uma explicação para frases como "pela segurança que o Comprasnet oferece” identificadas na questão aberta destinada a captar outros fatores motivadores de uso.

De acordo com Rosenfield \& Morville (1998, p. 4), é "odioso" para o usuário não conseguir encontrar no site web uma informação que ele sabe que está lá. Posto assim, verifica-se a importância do projeto de arquitetura da informação do site web, que tem como um de seus objetivos facilitar o entendimento da forma como as informações são estruturadas no site web, de maneira que permita ao usuário, intuitivamente, acessar as informações que deseja. Esta característica parece ser relevante para o usuário do Comprasnet, uma vez que $84 \%$ da amostra identificaram este fator como determinante de sua opção pelo uso do Comprasnet.

O fator efeito visual agradável relaciona-se com a estética do site web, materializada por meio da sua programação visual ou projeto multimídia do site web. De uma maneira geral, embora este conceito esteja presente em grande parte das listas de avaliação de sites web, a importância ou grau de relevância atribuído a este critério varia de acordo com a perspectiva de cada autor. Para Abels, White \& Hahn (1998), por exemplo, este critério ocupa o décimo quinto lugar em termos de importância atribuída pelo usuário. Todavia, a compilação de critérios de avaliação de sites web de Smith (1997) apresenta este critério em primeiro lugar. De acordo com Rosenfield \& Morville (1998, p. 5), os usuários são motivados, entre outras razões, pela estética do site web. Isto significa que pessoas podem usar um site web simplesmente pelo fato de este site web parecer-lhe esteticamente agradável. Isto parece refletir a opinião dos usuários do Comprasnet, uma vez que, na amostra pesquisada, este fator ocupa o terceiro lugar na freqüência de ocorrência como determinante da opção do usuário pelo uso do Comprasnet.

A representação da atualidade da informação disponibilizada pelo site web pode ser identificada pelo usuário mediante a verificação de aspectos como data da última atualização do site web como um todo, data da última atualização da página web específica que está sendo disponibilizada; ou, caso existam pesquisas em bancos de dados on-line, data referente à última atualização do banco de dados pesquisado; indicativos de freqüência de atualização das informações disponibilizadas no site web, estatísticas; entre outros. Este cuidado visa a garantir, conforme propõe Harris (1997) "uma fonte que seja correta hoje (não ontem)", ou ainda, segundo Alexander \& Tate (1999, p. 45), "indicações que permitam que o material disponibilizado seja percebido pelo usuário como atual".

Esta percepção da atualidade da informação parece estar presente no caso pesquisado, uma vez que as informações na base de dados do Comprasnet são atualizadas diariamente e também as datas da última atualização, bem como a freqüência de atualização das informações disponibilizadas no Comprasnet, que é indicada em sua página web anfitriã.

Para o usuário do Comprasnet, a impressão satisfatória das telas parece ter prioridade maior, no sentido de determinar o uso do site web, do que o comumente encontrado na literatura. Entretanto, deve ser considerada, nesta análise, a natureza fatual das informações disponibilizadas pelo Comprasnet. Algumas informações disponíveis no Comprasnet podem, inclusive, respaldar decisões sobre compras governamentais, por ser um site web oficial do governo federal, o que torna possível que as impressões de algumas de suas telas sejam consideradas como documentos que podem ser utilizados de forma oficial. Este fato pode explicar a diferença da importância da impressão satisfatória de telas para o usuário do Comprasnet.

Em termos de coerência dos links, por ser a criação de links entre recursos web uma característica natural à linguagem de hipertexto, é aconselhável a avaliação de cada link em relação à coerência do mesmo com a proposta do site web como um todo, uma vez que, pela 
facilidade de implementação de ligações entre recursos web, pode ser tentador incluir ligações entre recursos web que não são lógica ou conceitualmente compatíveis entre si. $\mathrm{O}$ conceito de coerência dos links também está relacionado, de forma geral, à expectativa de solução de um problema que motiva o usuário a acessar um site web. Isto quer dizer que o usuário pode criar o hábito de acessar um determinado site web que lhe oferece um link para uma outra funcionalidade, sendo que seu objetivo principal era acessar a funcionalidade, fazendo ela parte do site web acessado ou não. Isto pôde ser identificado a partir das afirmativas dos usuários ao alegarem o acesso ao Sistema Integrado de Cadastramento de Fornecedores (Sicaf) como um "outro motivo" para acessar o Comprasnet.

O conceito de inexistência de links quebrados está relacionado à frustração que acompanha o usuário ao clicar em um link quebrado, e este resultado no contexto do Comprasnet torna aconselhável a avaliação de cada link em relação à consistência do mesmo, principalmente se este link fizer referência a um recurso web que possua um outro endereço de rede.

Para Abels, White \& Hahn (1997), o conceito de comodidade para encontrar a informação está relacionado à dificuldade percebida pelo usuário ao buscar o mesmo tipo de informação em outras fontes. De uma maneira geral, diferentes usuários identificaram como "comodidade" suas respostas na alternativa "outros motivos" que influenciaram sua opção pelo uso do Comprasnet, em frases como "comodidade para participar de licitações", ou "comodidade para participar de pregões", "agilidade", "praticidade", entre outras que podem sugerir que não apenas a comodidade para acessar a informação influencia a opção pelo uso do Comprasnet, mas também a comodidade para "resolver um problema", de forma rápida e on-line, o que é definido por Combers (1995) como o principal objetivo do usuário da web.

Para Rosenfield \& Morville (1998, p. 8), o período de atenção do usuário da web é tão curto, que os autores chegam a qualificá-lo como "mítico", o que justifica a preocupação por se manter um site web cujas páginas web respondam à demanda de utilização de maneira a permitir que o usuário não se frustre por ter de esperar indefinidamente a manifestação de um recurso web, pois isto pode levá-lo a desistir daquele acesso.

O fator "tempo de resposta" foi identificado por $56 \%$ da amostra pesquisada como motivador de uso, representando o décimo primeiro critério na escala desta pesquisa. Em primeira análise, pode-se admitir que o "tempo de resposta" em relação aos outros fatores analisados não seja considerado pelo usuário como prioritário. Este fato, todavia, contraria alguns autores, que defendem que o usuário, ao acessar a web, tem não só a expectativa de solucionar um problema, mas sim de solucioná-lo rapidamente, o que, segundo Lindroos (1997), pode levá-lo à busca de outros sites web em ferramentas de busca, ou, caso a indisponibilidade aconteça de forma continuada, pode levar o usuário a perder a credibilidade no site web em questão.

O fato de o tempo de resposta não ter sido considerado pelos usuários do Comprasnet, entre os primeiros fatores determinantes de uso, pode indicar que isto não foi percebido pelo usuário como um problema. A explicação para esta percepção pode estar ligada ao fato de o Comprasnet corresponder às expectativas de seus usuários em relação ao tempo de resposta de suas páginas web, o que pode levar o sujeito a perceber o tempo de resposta como um "produto embutido" (utilizando-se o vocabulário de marketing), ou, simplesmente, uma característica já esperada do site web que apenas seria notada pelo usuário em sua ausência.

No caso do Comprasnet, por manter serviços, como o pregão eletrônico, que exigem resposta em tempo real, foi planejada uma arquitetura computacional superdimensionada para a demanda esperada. O Comprasnet está baseado em uma plataforma que opera em condições normais com menos de 50\% de sua capacidade de processamento em horários de pico, segundo os técnicos de hardware do Serpro. Em relação à largura de banda de rede disponível e utilizada pelo Comprasnet, segundo os técnicos de rede do Serpro, o Comprasnet utiliza até 40\% de sua capacidade disponível, também em horários de pico. De acordo com NTT (2001, p. 4-29), utilizando-se a teoria das filas, pode-se calcular o tempo de resposta de um sistema computadorizado a partir da mensuração da taxa de utilização das máquinas servidoras. Aplicando-se a fórmula apresentada em NTT (2001, p. 4-22) para as máquinas servidoras do Comprasnet, obteve-se o valor 0,4 (que representa 40\% de capacidade computacional utilizada), valor condizente com os declarados pelos técnicos do Serpro.

Partindo-se do princípio de que um sistema computadorizado pode apresentar desempenho satisfatório, desde que o valor obtido seja menor que um, que corresponde a 100\% de utilização das máquinas, estes dados permitem afirmar que o tempo de resposta do Comprasnet pode ser considerado adequado à demanda. Isto pode confirmar a suposição de que o tempo 
de resposta do Comprasnet seja percebido pelo usuário como um produto embutido.

Em relação aos outros fatores, situados abaixo do centro de gravidade da amostra, importa lembrar que a percepção do usuário do Comprasnet quanto à facilidade de navegação parece depender mais da facilidade de entendimento da estrutura do web do que de outros aspectos inerentes à navegabilidade e usabilidade. De acordo com os dados coletados, a facilidade de navegação representa, em ordem de freqüência de ocorrência, o décimo quarto critério na escala nesta pesquisa. Isto parece respaldar a consideração de que, uma vez que a estrutura do site web tenha sido percebida pelo usuário do Comprasnet como de fácil entendimento, ou, dito de outro modo, uma vez que o usuário se acostume com a forma como o site web está estruturado, também vai percebê-lo como de fácil navegação.

Para Abels, White \& Hahn (1998), apesar de a existência de mecanismo de busca no site web influenciar a opção pelo uso de um site web, caso este mecanismo de busca não seja efetivo, é preferivel que não seja disponibilizado. Em última análise, estes dados podem indicar que, para o usuário do Comprasnet, a facilidade de entendimento da estrutura do site web parece ter maior influência no seu processo de tomada de decisão de uso do site web do que a existência e efetividade do mecanismo de busca, a inibição de figuras, a objetividade da escrita e a ausência de uso gratuito de efeitos de multimídia.

\section{CONCLUSÃO}

Este estudo de caso foi motivado pela busca do conhecimento de quais seriam os critérios relativos a um site web que levariam um indivíduo a se tornar seu usuário, partindo-se do pressuposto de que a perspectiva do usuário seria importante para melhor estruturação do site web.
Foi identificada a necessidade de outros dados detalhados do perfil do usuário para o desenvolvimento de estudos que pretendam explorar em profundidade questões sobre como a percepção de cada critério pode ser afetada pelo conhecimento computacional do usuário.

Os resultados obtidos por esta pesquisa permitem o estabelecimento de subsídios para o planejamento e desenvolvimento de funcionalidades que pretendam ser centradas no usuário. Pode-se sugerir, para aqueles que forem implementar novas funcionalidades ou alterar as funcionalidades existentes no Comprasnet, que considerem, ao planejar ou desenvolver tais funcionalidades, os fatores motivadores de uso identificados por este trabalho.

Todavia, enquanto limitadas à comprovação por um estudo de caso, as ponderações e observações quanto aos resultados desta pesquisa não podem ser consideradas válidas para toda a web. Porém, estes resultados podem ser utilizados para comparações em trabalhos similares. Entre os estudos que podem vir a utilizar os resultados desta pesquisa para confrontá-los ou complementá-los, incluem-se pesquisas sobre tipologia de sites web, avaliação de sites web, estudos de uso e usabilidade de sites web, bem como estudos de usuários e estudos sobre sistemas centrados no usuário.

Finalmente, esta pesquisa possibilitou a identificação da percepção do usuário do Comprasnet em relação a web. Foi possivel estabelecer um conjunto de critérios que refletem a maneira como o usuário do Comprasnet avalia o site web, o que permite afirmar que o conjunto de critérios estabelecido pode possibilitar a avaliação de outro site web, portanto esta metodologia de pesquisa pode ser adaptada a outros contextos.

Artigo recebido em 09-02-2004 e aceito para publicação em 24-04-2004. 


\section{Tatiana de Almeida Furquim}

\section{REFERENCIAS}

ABELS, E. G.; WHITE, M. D.; HAHN, K. Identifying user-based criteria for web pages. Internet Research: Electronic Applications and Policy, v. 7, n. 4, p. 252-262, 1997

A user-based design process for web sites. Internet Research: Electronic Applications and Policy, v. 8, n. 1, p. 39-48, 1998.

ALEXANDER, J.; TATE, A. T. Web wisdom: how to evaluate and create information quality on the web. New Jersey : LEA, 1999. 156 p.

BERGHEL, H. et al. Cyberbrowsing: information customization on the web. Journal of the American Society for Information Science, v. 50, n. 6, p. 505-513, 1999

BERNERS-LEE, T. Weaving the web: the original design and ultimate destiny of the world wide web. San Francisco : HarperCollins, 1999. 219 p.

BEVAN, N. Usability issues in web site design. Disponível em: <http:// www.npl.co.uk/npl/sections/us>. Acesso em: 25 nov. 2000.

CIOLEK, T. M. The six quests for the electronic grail: current approaches to information quality in WWW resources. Review Informatique el Statistique dans les Sciences Humaines, Lieg, Belgian, v. 14, p. 45-71, 1996. Disponível em: <http://www.ciolek.com/PAPERS/ QUEST/QuestMain.html>. Acesso em: 17 ago. 2000.

HARRIS, R. Evaluating Internet research sources. Disponível em: <http://www.virtualsalt.com/evalu8it.htm>. Acesso em: 10 set. 2001.

KOTLER, P. Administração de marketing. São Paulo : Atlas, 1996. $676 \mathrm{p}$.

LINDROOS, K. Use quality and the world wide web. Information and Software Tecnology, v. 39, n. 12, p. 827-836, Dec. 1997.
MORKES, J.; NIELSEN, J. Concise, scannable and objective : how to write for the web. Disponivel em: <http://www.useit.com>. Acesso em: 17 maio 2000

NIELSEN, J. Top ten mistakes of web management. Disponível em: <http://www.useit.com>. Acesso em: 10 set. 2001.

NIPPON TELEGRAPH AND TELEPHONE EAST CORPORATION. System performance design. Okinawa, Japão, 2001. Paginação irregular.

RICHARDSON, R. J. Pesquisa social: métodos e técnicas. 3. ed. São Paulo : Atlas, 1999. 334 p.

ROSENFIELD, L.; MORVILE P. Information architecture for the world wide web: designing large-scale web sites. Cambridge : O'Reilly, 1998. 197 p.

SHNEIDERMAN, B. Designing information abundant web sites: issues and recommendations. International Journal of Human-Computer Studies, v. 47, n. 1, p. 5-29, 1997.

SMITH, A. G. Testing the surf: criteria for evaluating internet information sources. Public-Access Computer Systems Review, v. 8, n. 3, 1997. Disponível em: <http://info.lib.uh.edu/pr/v8/n3/ smit8n3.html>. Acesso em: 21 set. 2001.

SWEETLAND, J. H. Reviewing the world wide web: theory versus reality. Library Trends, v. 48, n. 4, p. 748-768, Spring 2000.

TROCHIM, W. Evaluating websites. Disponível em: <http://trochim.human.cornell.edu/WebEval/webintro/ webintro.htm>. 